I N S T I T U T O

DE

M E D I C I N A

T R O P I C A L

DE

SÃO PAULO

JOURNAL OF THE SÃO PAULO INSTITUTE OF TROPICAL MEDICINE

1Hospital General "Dr. Agustín O’Horán”, División de Pediatría, Mérida, Yucatán, México

2Universidad Autónoma de Yucatán, Facultad de Medicina, Unidad Interinstitucional de Investigación Clínica y Epidemiológica, Mérida, Yucatán, México

${ }^{3}$ Asociación Colombiana de Infectología, Comité de Medicina Tropical, Zoonosis y Medicina del Viajero, Bogotá, Colombia

${ }^{4}$ Universidad Autónoma de Yucatán, Centro de Investigaciones Regionales Dr. Hideyo Noguchi, Laboratorio de Enfermedades Emergentes y Reemergentes, Mérida, Yucatán, México

${ }^{5}$ Hospital General “Dr. Agustín O’Horán”, Departamento de Patología, Mérida, Yucatán, México

Correspondence to: Karla Rossanet DzulRosado

Universidad Autonoma de Yucatan, Campus de Ciencias de la Salud, Centro de Investigaciones Regionales Dr. Hideyo Noguchi, Avenida Itzáes, № $490 \times$ Calle 59, Col. Centro, CP 97000, Merida, Yucatan, Mexico

Tel: +52 999 924-5755 F: 1151

E-mail: karla.dzul@correo.uady.mx

Received: 26 August 2020

Accepted: 18 November 2020

\section{Fatal murine typhus with hemophagocytic lymphohistiocytosis in a child}

\author{
Victor Federico Leal-López ${ }^{1}$, Juan J. Arias-León ${ }^{2}$, Álvaro A. Faccini-Martínez ${ }^{(1)}$, \\ Cesar Lugo-Caballero ${ }^{4}$, Carlos Quiñones-Vega ${ }^{1}$, José M. Erosa-Gonzalez ${ }^{5}$, \\ Karla Rossanet Dzul-Rosado ${ }^{(14}$
}

\section{ABSTRACT}

Hemophagocytic lymphohistiocytosis is a rare complication in Rickettsia typhi infections. We report the case of a 2-year-old boy with sudden night-onset fever, pallor, neck adenopathy and erythematous macular rash on the thorax, thighs and buttocks. During admission, he developed hyponatremia, hypoalbuminemia, anemia, thrombocytopenia, leukopenia, neutropenia, liver damage, hemorrhages and persistent fever. No hematological improvement was observed after the initial management, neoplastic diseases were discarded by bone marrow aspiration and lymph node biopsy; hemophagocytic lymphohistiocytosis was diagnosed. By immunohistochemistry and indirect immunofluorescence, murine typhus was also diagnosed and doxycycline was started with transitory recovery. Later, the child developed kidney failure and distributive shock that evolved to cardiac arrest and death. This is the first case report in Mexico on a fatal murine typhus associated with hemophagocytic lymphohistiocytosis in which the etiology was evidenced by histopathology.

KEYWORDS: Murine typhus. Rickettsia typhi. Immunohistochemical staining. Hemophagocytic lymphohistiocytosis.

\section{INTRODUCTION}

Rickettsiae of the typhus group (Rickettsia typhi and Rickettsia prowazekii) are transmitted by fleas and clothes lice, respectively ${ }^{1}$. In Mexico, Murine Typhus (R. typhi) is the second most frequent rickettsial disease, with 201 cases in 2018 and 2019, while Rocky Mountain Spotted Fever (R. rickettsii), and unspecified rickettsial disease accounted other 617 and 294 cases, respectively. General clinical features of rickettsioses include fever, headache, malaise, exanthema, arthralgia, myalgia, nausea or vomiting, and can evolve to distributive shock and multiple organ failure. Rickettsioses of the spotted fever group (SFGR) and rickettsioses of the typhus group (TGR) show some differences in presentation and severity. Patients with murine typhus usually present with a severe but nonspecific febrile illness, with rash in approximately half of the cases, more commonly as fine erythematous papules on the abdomen spreading centrifugally to the trunk and extremities, but often sparing the face, palms and soles. In the case of the spotted fever group, the rash is characteristic and presents as small red spots of blotches that begin on the writs, palms and soles, then spreads to the arms, legs an torso without itch, and tends to appear 3 to 5 days after the onset of fever ${ }^{2}$. Hemophagocytic lymphohistiocytosis (HLH) accounts for diverse etiologies, primary genetic HLH is caused by a mutation in a component of the perforin-mediated cytotoxicity 
pathway and may be either familial or associated with genetic immunodeficiency syndromes (Chediak-Higashi, Griscelli type-2, X-linked lymphoproliferative disorder); and acquired, usually associated with autoimmune, oncologic, infectious or other syndromes. HLH is a disorder characterized by a wide array of life-threatening conditions secondary to immune dysregulation with high morbidity and mortality, and management includes immune modulation, immunosuppression and chemotherapy ${ }^{3-7}$. Few cases of HLH have been reported secondary to rickettsial diseases, and our case is the $6^{\text {th }}$ patient with this condition reported in the literature so far ${ }^{8-10}$. Herein, we describe a patient with fatal $\mathrm{HLH}$, diagnosed as murine typhus through serology and immunohistochemical (IHC) staining.

\section{CASE REPORT}

We present the case of a 2-year-old boy, whose father had fever and exanthema one month before the onset of the child's disease that remained undiagnosed. The child had a normal weight at birth, was breastfeed for six months and his vaccination was up to date. The family reported contact with two dogs in the house. No other relevant information was retrieved.

The first symptom was a sudden fever $\left(38^{\circ} \mathrm{C} / 100.4^{\circ} \mathrm{F}\right)$ that appeared on November 02, 2015, that was most frequent at nights and persisted during the entire disease period. The boy received nimesulide and amoxicilin. Three days later, the child developed pallor. On day 5, a blood test was performed finding leucopenia (leucocytes 2,100 cel/ $\mu \mathrm{L}$ [normal range for age is $6-17 \times 10^{3} / \mu \mathrm{L}$ ]), anemia (hemoglobin $8.9 \mathrm{~g} / \mathrm{dL}$ [minimun for age is $12.5 \mathrm{~g} / \mathrm{dL}$ ]) and thrombocytopenia $\left(75 \times 10^{3} / \mathrm{mL}\right.$ [normal range for age is $\left.150-350 \times 10^{3} / \mathrm{mL}\right]$ ), leading to the admission of the patient on November 06, 2015.

On November 08, the second day after admission, additional blood tests were performed finding hypoalbuminemia $(2.9 \mathrm{~g} / \mathrm{dL}$ [normal 3.4 to $5.4 \mathrm{~g} / \mathrm{dL}$ ]), high liver enzymes (AST $1333 \mathrm{U} / \mathrm{L}$ [normal for age 0-40 U/L], ALT $972 \mathrm{U} / \mathrm{L}$ [normal for age 0-30 U/L], alkaline phosphatase $753 \mathrm{U} / \mathrm{L}$ [normal range for age is $145-420 \mathrm{U} / \mathrm{L}$ ], LDH 1,714 U/L [normal range for age is 60-170 U/L], prolongation of coagulation times (PT $16.6 \mathrm{~s}$ [normal for age 12.1-14.5 s], PTT $54.5 \mathrm{~s}$ [normal for age $33.6-43.8 \mathrm{~s}])$, hyponatremia ( $126 \mathrm{mEq} / \mathrm{L}$ [normal range $135-145 \mathrm{mEq} / \mathrm{L}]$ ) and elevation of the $\mathrm{C}$ reactive protein $(2.4 \mathrm{mg} / \mathrm{dL}$ [normal $<0.5 \mathrm{mg} / \mathrm{dL}])$. Multiple enlarged lymph nodes were found in the neck (hard, unmovable, cold and unpainful, measuring $10 \mathrm{~mm}$ in diameter). Other findings included hepatomegaly and splenomegaly (4 and $5 \mathrm{~cm}$ respectively), erythematous, macular, non-itching rash on the thorax thighs and buttocks, and a single left inguinal adenopathy.

During the first week after admission, the fever kept appearing on a daily basis with nocturnal and needle pattern, associated with coughs without consolidations or radiological images suggestive of pneumonia. Bone marrow aspiration was performed and results were non-conclusive, but the presence of activated lymphocytes without signs of leukemia were observed. From the routine blood tests performed, the most relevant results were the persistence of cytopenia in three cellular lines manifesting as anemia (normocytic, normochromic, $\mathrm{Hb} 6.3 \mathrm{~g} / \mathrm{dL}$ [minimun for age $12.5 \mathrm{~g} / \mathrm{dL}$ ]), deep neutropenia $\left(0.3 \times 10^{3} / \mu \mathrm{L}\right.$ [normal range for age is $\left.1.5-10.8 \times 10^{3} / \mu \mathrm{L}\right]$ ), and thrombocytopenia $\left(98 \times 10^{3} / \mathrm{mL}\right.$ [normal range for age is $\left.\left.150-350 \times 10^{3} / \mathrm{mL}\right]\right)$. The kidney function was normal at admission without prejudice to glomerular filtration at this stage (GF $88 \mathrm{~mL} / 1.74 \mathrm{~m}^{2} \mathrm{SC} / \mathrm{min}$ by modified Schwartz equation). Clinically, the boy evolved with a hemorrhagic syndrome managed with multiple transfusions. Hyponatremia was persistent since day $1(126-128 \mathrm{mEq} / \mathrm{L}$ [normal range is $135-145 \mathrm{mEq} / \mathrm{L}])$. During the course of the disease, a liver failure was detected with incoagulable prothrombin time and persistent hypoalbuminemia. Lactate dehydrogenase peaked over $6700 \mathrm{UI} / \mathrm{L}$ [normal range for age is $60-170 \mathrm{U} / \mathrm{L}$ ] with a later drop, without remission of the hepatic failure. Serological tests were performed for HIV, Epstein-Barr virus, Hepatitis A, B and C virus, Toxoplasma gondii, cytomegalovirus, rubella virus, herpes simplex, Treponema pallidum, as well as the Weil-Felix reactions, all of them yielding negative results.

In the second week, a new bone marrow biopsy was performed, which showed cellularity of $70 \%$, inverted myeloid-erythroid ratio from 1 to 1.5 , myeloid hypoplasia with focal maturation to bands, immature forms and erythroid series hyperplasia with megaloblastic maturation. Megakaryocytic series presented megakaryocytes and hypolobulated nuclei, histiocytes with hemophagocytosis and $1 \%$ of lymphocytes (Figure 1A); considering these results, leukemia was ruled out. A lymph node biopsy was also performed, showing ganglionic sinus partially infiltrated by histiocytic cells, with a total loss of architecture and some hyperplasic lymphoid follicle (Figure 1B). Immunohistochemistry for Langerhans cells histiocytosis was performed, as well as long bones, skull and thorax radiographs, ruling out Langerhans disease.

During the third week after admission, fever was persistent and jaundice developed, whereby doxycycline was empirically initiated at a dose of $2.2 \mathrm{mg} / \mathrm{kg} /$ day orally. Remission of fever was observed within the first $48 \mathrm{~h}$ of doxycycline with improvement of the general condition. 

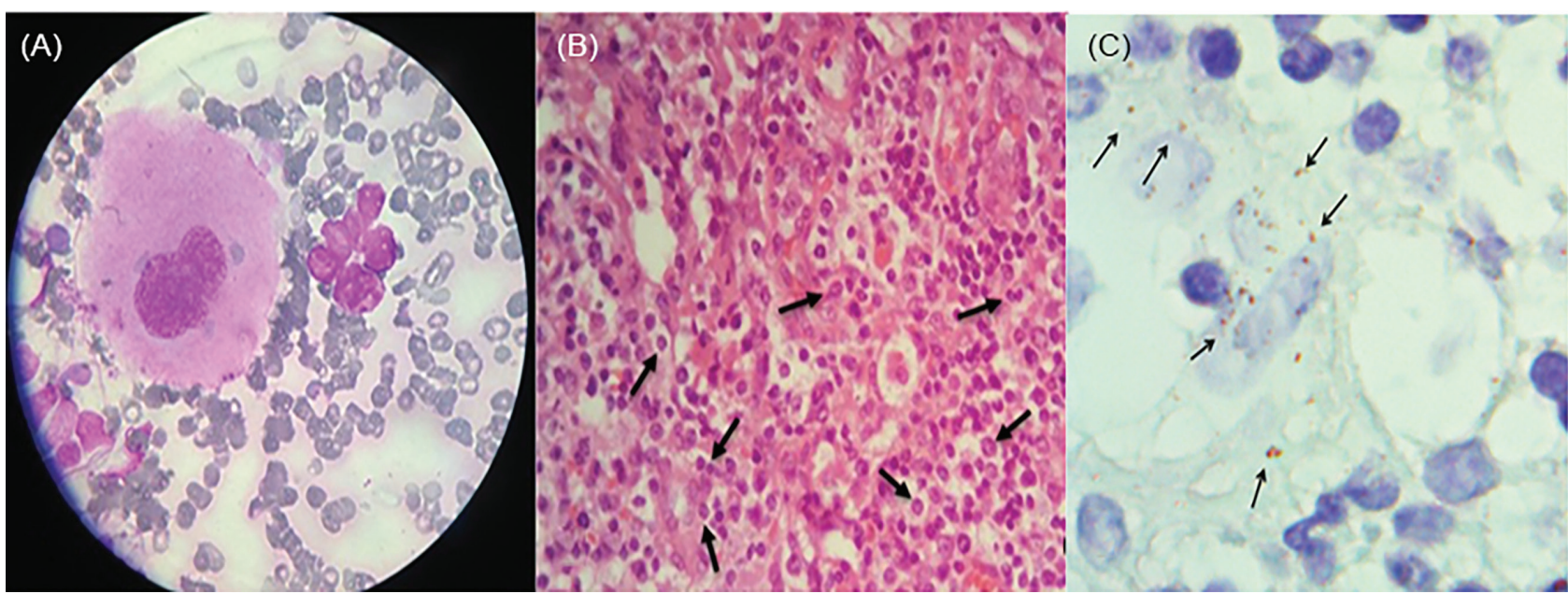

Figure 1 - Histopathological results: (A) Bone marrow biopsy: cellularity $70 \%$, inverted myeloid-erythroid ratio from 1 to 1.5 , myeloid series hypoplasia with focal maturation up to bands with immature forms and erythroid series hyperplasia with megakaryoblast maturation, megakaryocytic series with micro-megakaryocytes and hypolobulated nuclei, histiocytes with hemophagocytosis and $1 \%$ lymphocytes; (B) Lymph nodule biopsy: partial ganglionic sinusoid infiltration with histiocyte-like cells, with almost all the architecture loss and some hyperplasic lymphatic follicles; (C) Immunohistochemistry evaluation for Rickettsia: Post-mortem, 3 mm paraffined histological sections analyzed by a modified immunoperoxidase technique, hyperimmune mouse anti-Rickettsia antibodies in a 1:500 dilution, yielding a positive result. Original magnification x100.

Later on, on days 3-5 after the introduction of doxycycline, new fever peaks, jaundice, and asthenia reappeared, accompanied by increased triglycerides, persistence of cytopenia and low fibrinogen levels. At this stage, Leptospira interrogans infection was ruled out by a negative polymerase chain reaction. After ruling out malignancy, the presence of hemophagocytes in bone marrow and considering the presence of a confirmed splenomegaly, the diagnosis of hemophagocytic lymphohistiocytosis was established and steroids management was started (methylprednisolone $30 \mathrm{mg} / \mathrm{kg} /$ day). At this stage, 20 days after the onset of symptoms, DNA was extracted from a blood sample to perform a nested polymerase chain reaction for the 17-kDA gene of Rickettsia spp. with a negative result. In-house indirect immunofluorescence assay for IgM and IgG against Rickettsia rickettsii and Rickettsia typhi were performed, yielding a first negative result on day 14 after the onset of symptoms and seroconversion on day 27, up to a titre of 1:128 for $\operatorname{IgM}$ and 1:512 for $\operatorname{IgG}$ only to Rickettsia typhi.

Over the fourth week after admission, the patient developed a hemorrhagic syndrome with persistence of low fibrinogen levels associated with petechiae and gingivorrhagia. The patient was admitted to the Pediatric intensive care unit due to neurological and respiratory deterioration. Antimicrobial management at this point had escalated to Meropenem, vancomycin and amphotericin B, with a nosocomial sepsis being suspected, although blood cultures were all negative. Doxycycline was suspended after seven days due to gastric bleeding. After five days on methylprednisolone, dexamethasone was also added, with a poor response. A new abdominal ultrasound reported diffuse hepatic and kidney damage with an altered cortexmarrow relationship and a hyperechoic pattern, and ascites was also reported. The urine output decreased in the last $24 \mathrm{~h}$ of life, with neurological and hemodynamic sudden worsening. Mechanical ventilation and vasopressor drugs were started, then alveolar diffuse hemorrhage and anuria developed. The patient died on day 35 of hospitalization, 41 days after the onset of fever.

A post-mortem histopathological test was performed on a lymph node biopsy. Histological sections of the lymph node were analyzed by Immunohistochemistry (IHC) by means of a modified immunoperoxidase technique for paraffin-embedded tissues ${ }^{11}$. A positive result was obtained using a polyclonal mouse hyperimmune antiserum directed to a conserved region of the OmpB sequence among rickettsiae of the spotted fever and typhus groups ${ }^{12}$ (Figure 1C).

\section{DISCUSSION}

Decreasing the spread of rickettsioses and management guidelines can improve the time of diagnoses and reduce complications. We must emphasize that a first negative immunofluorescence test result does not rule out the disease, and a negative PCR after the $3^{\text {rd }}$ day of fever has also a very low sensitivity. According to CDC and WHO, seroconversion of immunoglobulins is still the gold standard for the diagnosis of rickettsial diseases ${ }^{2,13}$. 
Standard therapy for rickettsial diseases in America is doxycycline $(4.4 \mathrm{mg} / \mathrm{kg} / \mathrm{day}$ divided into two doses), chloramphenicol is still a second choice, but in some regions with high experience in rickettsial diseases such as India and Indonesia, the use of azithromycin, levofloxacin or an association of doxycycline are recommended according to the length and severity of symptoms. In critically ill patients, maximum doses must be sustained and adjusted according to liver and kidney functions. The use of tetracyclines in children is considered safe in all age groups ${ }^{14,15}$.

Our patient received enteral doxycycline even though bleeding from the upper digestive tract, since we had no intravenous formulation, and this may have affected the blood levels of antimicrobial agents, as well as the duration of treatment that lasted seven days and was discontinued three days after remission of the fever, but fever came back shortly after doxycycline was discontinued ${ }^{13,15}$.

Complications are present in approximately $26 \%$ of patients with murine thypus; the most frequent are pulmonar ones (pneumonia, pulmonary effusion and respiratory failure), followed by central nervous system involvement including altered level of consciousness, seizures and ataxia. Other common complications include kidney injury, abnormal ocular findings and septic shock $^{13}$. Hemophagocytic lymphohistiocytosis is a rare complication, but due to the high mortality rates it should be suspected in order to start treatment before the damage is irreversible. The case we presented fulfilled five criteria according to the Histiocyte Society, thus justifying the use of steroids during the late phase of the disease. Treatment with immunomodulators is recommended in cases with no response to initial management ${ }^{16}$. In our case, since it was not possible to rule out a primary cause of Hemophagocytic lymphohistiocytosis caused by genetic defects, among others, this fact could be responsible for the disease progression despite adequate etiological treatment with doxycycline. Additionally, the specific HLH therapy was used in the initial stages when the patient became critically ill. It is noticeable that the delay to start doxycycline treatment of up three weeks after the onset of symptoms could be one of the factors that allowed the HLH to be fatal, so that an earlier specific treatment should have improved the outcome of the patient.

Rickettsiosis should be suspected in all patients with bone marrow failure and histiocytes on biopsy, since cases associated with Rickettsia conorii and Rickettsia rickettsii have already been described and we are now presenting the sixth case of Rickettsia typhi with HLH diagnosed by IFA and IHC staining. This technique can be useful in the early diagnosis of these pathologies before the rise in immunoglobulins takes place, anticipating the diagnosis and thus avoiding many deaths due to Rickettsia in Yucatan that are overlooked, not confirmed or not reported ${ }^{17,18}$.

\section{ACKNOWLEDGMENTS}

We thank Dr. Erick Gutierrez Juárez for his valuable contribution in this case report.

\section{FUNDING}

CONACYT project SALUD-2015-2-261885 financed the diagnostic tests used in this case report.

\section{REFERENCES}

1. Nawab T, Srinivasa S, Reddy SP. A clinical study of rickettsial disease and its manifestations. Curr Pediatr Res. 2015:19:1720.

2. Biggs HM, Behravesh CB, Bradley KK, Dahlgren FS, Drexler NA, Dumler JS, et al. Diagnosis and management of tickborne rickettsial diseases: Rocky Mountain spotted fever and other spotted fever group Rickettsioses, Ehrlichioses, and Anaplasmosis - United States. MMWR Recomm Rep. 2016:65:1-44.

3. Esteban YM, de Jong JL, Tesher MS. An overview of hemophagocytic lymphohistiocytosis. Pediatr Ann. 2017: 46:e309-13.

4. Rouphael NG, Talati NJ, Vaughan C, Cunningham K, Moreira $\mathrm{R}$, Gould C. Infections associated with haemophagocytic syndrome. Lancet Infect Dis. 2007;7:814-22.

5. Cascio A, Pernice LM, Barberi G, Delfino D, Biondo C, Beninati $\mathrm{C}$, et al. Secondary hemophagocytic lymphohistiocytosis in zoonoses: a systematic review. Eur Rev Med Pharmacol Sci. 2012;16:1324-37.

6. Cascio A, Giordano S, Dones P, Venezia S, Iaria C, Ziino O. Haemophagocytic syndrome and rickettsial diseases. J Med Microbiol. 2011;60:537-42.

7. Karra N, Dolinski R, Akria L, Yampoulski Y, Awad J. A case of hemophagocytic lymphohistiocytosis associated with Mediterranean spotted fever in a healty 29 -year-old female. Open Forum Infect Dis. 2020;7:ofaa355.

8. Walter G, Botelho-Nevers E, Socolovschi C, Raoult D, Parola P. Murine typhus in returned travelers: a report of thirty-two cases. Am J Trop Med Hyg. 2012;86:1049-53.

9. Iaria C, Colomba C, Di Carlo P, Scarlata F, Tolomeo M, Cascio A. Rickettsia typhi and haemophagocytic syndrome. Am J Trop Med Hyg. 2017;97:1632.

10. Iaria C, Colomba C, Di Carlo P, Scarlata F, Cascio A. Murine typhus and hemophagocytic syndrome. J Pediatr Hematol Oncol. 2018;40:493-4. 
11. Schriefer ME, Sacci Jr JB, Dumler JS, Bullen MG, Azad AF. Identification of a novel rickettsial infection in a patient diagnosed with murine typhus. J Clin Microbiol. 1994:32:94954.

12. Dzul-Rosado K, Balam-Romero J, Valencia-Pacheco G, Lugo-Caballero C, Arias-León J, Peniche-Lara G, et al.. Immunogenicity of OmpA and $\mathrm{OmpB}$ antigens from Rickettsia rickettsii on mononuclear cells from Rickettsia positive Mexican patients. J Vector Borne Dis. 2017;54:317-27

13. Tsioutis C, Zafeiri M, Avramopoulos A, Prousali E, Miligkos M, Karageorgos SA. Clinical and laboratory characteristics, epidemiology, and outcomes of murine typhus: a systematic review. Acta Trop. 2017;166:16-24.

14. Sandoval C, Vásquez B. Evaluación de reactividad inmunohistoquímica con método del Complejo AvidinaBiotina (ABC). Int J Med Surg Sci. 2016:3:909-18.
15. Kaore SN, Sharma P, Yadav VK, Sharma R, Kaore NM, Langade DG. Treatment update for scrub typhus. Ind Med Gaz. 2011:1-5.

16. Henter JI, Horne A, Aricó M, Egeler RM, Filipovich AH, Imashuku S, et al. HLH-2004: diagnostic and therapeutic guidelines for hemophagocytic lymphohistiocytosis. Pediatr Blood Cancer. 2007;48:124-31.

17. Espinosa Bautista KA, Garciadiego Fossas P, León Rodríguez E. Síndrome hemofagocítico. Conceptos actuales. Gac Med Mex. 2013;149:431-7.

18. Quintero Vélez JC, Hidalgo M, Rodas Gonzaléz JD. Rickettsiosis, una enfermedad emergente y re-emergente en Colombia. Univ Sci. 2012;17:82-99. 